\title{
Domots are coming ! or How to manage building automation in a balanced way?
}

\author{
Luís Gomes and A. Steiger-Garção \\ Universidade Nova de Lisboa, FCT, DEE and UNINOVA, CRI \\ Quinta da Torre, 2825 Monte de Caparica, Portugal \\ Fax: +351-1-2941253. E-mail:\{lugo,asg\}@uninova.pt
}

\begin{abstract}
One goal of the presented works is the development and exploitation of a building infrastructure allowing integration of services and activities. Two approaches coexist, related with the automatic versus anthropocentric issues. In one hand (the automatic way), activities inside buildings could be more organised, optimised, efficiently controlled and less expensive if a set of complementary infrastructures is available, acting in a cooperative way and enabling new specific functionality implementation. In the other hand, the anthropocentric view stresses the dependency on the occupant needs and wishes.

In this paper, it will be presented the definition of reference architectures and models that adequately supports the basic functionalities and their relations with building activities, as well the necessary infrastructure for a robust and open system based implementation, considering the occupant needs and wishes. Entities, named domots, that could manage some building resources and are able to dialogue and to be at the occupant disposal will be introduced $($ domots $=$ "domus" + "robot"). Already developed domots will be presented.
\end{abstract}

\section{Keywords}

Intelligent buildings, Distributed systems, System integration, Balanced automation

\section{INTRODUCTION}

Some years ago, apprehension on energy wasting associated with policies targeted to reduce costs with utilisation and maintenance of buildings has led to the concept of intelligent buildings (IB). This concept has been misunderstood and redefined several times. Undoubtedly, it is a multidisciplinary application area, where different expertise, from archicteture, building construction to systems and control engineering, could contribute in some complementary way for the benefit of the building occupants. 
One consequence of this leads to the definition of some concepts, like "building intelligence", in different and complementary ways, depending on the targeted community to be addressed by the presentation. It seems that is necessary to evaluate the "building intelligence" in terms of four basic elements (IBI, 1987): structure, systems, services and management.

This means that the "building intelligence" starts at the very beginning of the building's project and stands during the operation and exploitation of the building.

In the current framework, an intelligent building is a structured environment, equipped with several sensorial and control systems targeted for monitoring and control activities (either inside or outside the building), using heterogeneous network infrastructures enabling human and computer systems communications.

One goal of the presented works is the development and exploitation of such infrastructure allowing integration of services and activities. We argue that activities inside buildings could be more organised, optimised, efficiently controlled and less expensive if a set of complementary infrastructures is available, acting in a cooperative way and enabling the implementation of new specific functionalities. These new functionalities are obtained through a synergistic subsystems interaction, in order to obtain an improved activity support.

Let's pick up an example regarding aids to people searching and location inside a building (for telephone call routing, for example). In this case, personal transponders could be used in order to determine the location of some building occupant, through the use of an expensive dedicated system based on the detection of the different radio-frequency or infrared characteristics of the transponders. An alternative solution, could relies on the information provided by an already installed access control system and their direct use by the PABX in order to obtain a transparent follow-me in any call routing.

This means that, in a general way, adding sensors, actuators and electronic control just to do an optimal control of some feature of a process does not pay; it is necessary to integrate systems in the building to get global improvements, like better management and new functionalities and services. The way we can handle the different systems in order to get that is fundamental and is available through integration. So, it seems that the keyword is changing from Intelligence to Integration in the IB concept.

In a rough way, for a specific functionality, we may associate costs and the level of system integration as presented in figure 1 .

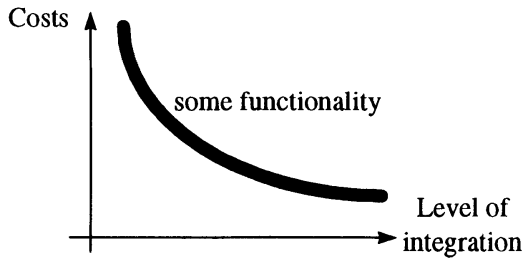

Fig. 1 - Dependency of the cost of some building functionality on the level of system's integration.

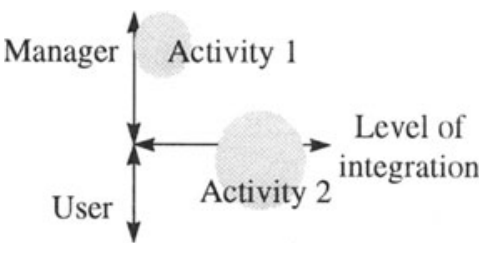

Figure 2 - Activity mapping.

It is also interesting to map into a chart, the level of coverability of some specific activity or functionality in terms of the level of integration needed and the target building occupant, e.g. a specific user or a resource manager. Consider figure 2 as an example, where activity 1 represents a very specific and isolated managing activity requiring a low level (or inexistence) 
of integration, while for activity 2 it is needed a high level of integration to achieve some goal interesting both for managers and common users (e.g. automatic follow-me call routing).

A complementary line of thought addresses the "automatic versus requested" issues. We strongly stress that this is a matter of major importance. This duality has to be solved through the occupant needs and wishes, through what could be characterised as a balanced way. This means that some functionality will only be active if the occupant is interested on it. Let's came back to the example; although there could be available an automatic follow-me functionality, the occupant has to have the opportunity to enable and to disable it whenever he or she wants.

In this paper, it will be presented the definition of reference architectures and models that adequately supports the basic functionalities and their relations with building activities, as well the necessary infrastructure for a robust and open system based implementation, considering the occupant needs and wishes. This is the core of a design methodology, enabling the development and integration of a set of tools and sub-systems for the design and implementation of the balanced and user friendly control and management of intelligent buildings.

\section{DOMOTS ARE WELCOME}

As already stated, the building is an heterogeneous environment composed by different autonomous and cooperative sub-systems and equipped with several sensors and control devices. In order to reach a full exploitation of such infrastructure it is mandatory to have a user-friendly support architecture enabling easy and efficient dialogues between different occupant and the building's infrastructure. These will take advantages of the infrastructure in order to get an improved activity support.

In this line of thought, it is necessary to have a set of entities responsible for those dialogues. In other words, some entities that could manage some resources and are able to be at the occupant disposal. We call those entities by the name of domot, originated by the aggregation of the words "domus" and "robot". Those entities have capabilities to: i) sense the building environment; ii) actuate on the building infrastructure; iii) communicate with the building occupants; iv) take decisions and actions according to the three former capabilities.

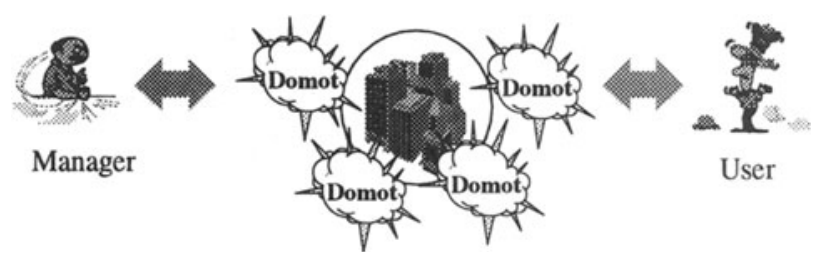

Figure 3 - A domot is always in the path of any user/manager.

Those capabilities could be achieved in an autonomous or non-autonomous way, i.e. to accomplish some goal the domot could rely on some external agent or other domot. It could be seen as an agent targeted for building occupant support. In a broad sense, a domot is any entity that could act in the building framework, making the interface between occupants and the building resources, supporting some functionality or activity.

As examples of already developed domots, we may pick up two examples. The first one is an application that produces telephone call traffic reports every month; the second one is an 
user interface application target for real-time monitoring of the building, showing the status of different devices, like presence detectors, cameras (images) and telephone terminals.

\section{MODELLING VIEWS}

As already stated, the intelligent building is a multidisciplinary concept; so, it is expected that different people involved in its design have different views of the building. From the point of view of this work, we have to exclude some views associated with construction, environmental and architectural issues and stress the views associated with the system and control engineering.

Anyway, we may consider two types of modelling objectives. In the first one, where specification is the goal and the model characteristics have to be expressed from an external point of view (i.e. in a black box like attitude), while in the second one, design/implementation is the goal and an internal description of the solution is pursued.

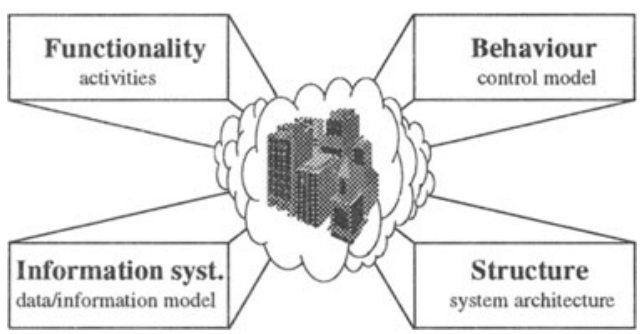

Figure 4 - Different views of the building.

Figure 4 presents the four main modelling views considered: behaviour, functionality, structure and information systems. They have to be considered as complementary views. The "functionality" view may be seen as an example of "modelling for specification", while the "behaviour" and "structure" views could be included in the "modelling to design" group.

We include in the "behaviour" view all the models associated with the control of the different devices and resources and in the "structure" view the models related with hardware and software architectures (refer to figure 4). In the next two sections more detailed presentations of these views will be done.

In the "functionality" view, we include the activity's descriptions, in terms of list of procedures or data-flow diagrams (refer to figure 4).

In the "information systems" view, we include the data/information models (refer to figure 4). The data model in use is composed by two types of data entities: the first one named "Instances" and the second one named "Catalogue". The last one stores general characteristics of all devices and equipment available or in use in the building. The former one contains specific description of all active devices and systems already installed.

\section{CONTROL MODELLING}

The use of discrete-event systems formalisms, like Petri nets (Murata, 1989) and statecharts (Harel, 1987), seem to be very adequate to model control for the different sub-systems in use. 
A synchronised coloured Petri net model proposed in (Gomes and Steiger-Garção, 1995b) is intended to be used for that purpose, extending developed small-sized academic examples.

Let's consider the example of a security system composed by an intrusion system and a CCTV system (Gomes and Steiger-Garção, 1994). At design time is important to specify the number of alarm situations, their definitions and associated actions. The formalism used here to describe such structures is Petri nets. This model could be used at control time also. Suppose we have a room with four sensors with binary outputs, e.g. sensor A (door-lock switch), sensor B (volumetric presence detector), sensor C (ultrasonic movement detector) and sensor D (infrared barrier detector). A simple rule to conclude about an alarm condition in the room is:

IF (sensorA OR (sensorB AND sensorC) OR sensorD) THEN alarmONlab1

The Petri net model representing such a rule is straightforward. Also, if the alarm structure is hierarchically organised, its net representation is easy. Consider the definition of an alarm in the lab's area through the rule

IF (alarmONlab1 OR alarmONlab2 OR alarmONlab3) THEN alarmONlabArea

Their global Petri net model is shown in figure 5, where the sensors are modelled by input transitions, that will be fired at the beginning of the treatment cycle (to enable formal validation, a place could model this state).

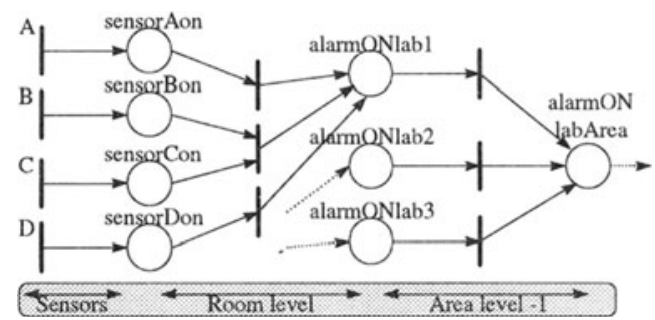

Figure 5 - A simplified Petri net model of an alarm structure.

This Petri net model is adequate to represent such sensor network control structures and could manage increasing complexity. For instance, if we intend to use some complex sensors, like presence detection based on image processing (sensorE) or person identification based on fingerprint analysis (sensorF), the same model could be used, adding these new dependencies. According to figure 5, it could be modelled just changing the references from $A$ to $E$ and from $\mathrm{D}$ to $\mathrm{F}$ (someone), where these values ( $\mathrm{E}$ and $\mathrm{F}$ (someone)) were the results of some dedicated processing. The parameter "someone" could be adequately modelled through coloured tokens, regarding the Coloured Petri net formalism (Jensen, 1990).

This net model could be adequately embedded into the user interface targeted to support surveillance activities. The developed prototype relies in a hierarchically organised plant of the institution facilities, including reference to all devices and systems installed. The plant may be divided into as many levels as necessary, as illustrated in figure 6 . The alarm structure defined and represented by the net of figure 5 , could be directly associated with this synoptic. The user may get specific information related with any device shown in the plant.

Furthermore, for the case of some continuous variable systems, like heating, ventilation and air conditioning, a discrete-event system approach could be used if we may substitute the mathematical model by a set of rules in order to construct a fuzzy controller. The modelling of 
fuzzy controllers and their integration into discrete-event system models using coloured Petri nets is possible and easy (Gomes and Steiger-Garção, 1995a).

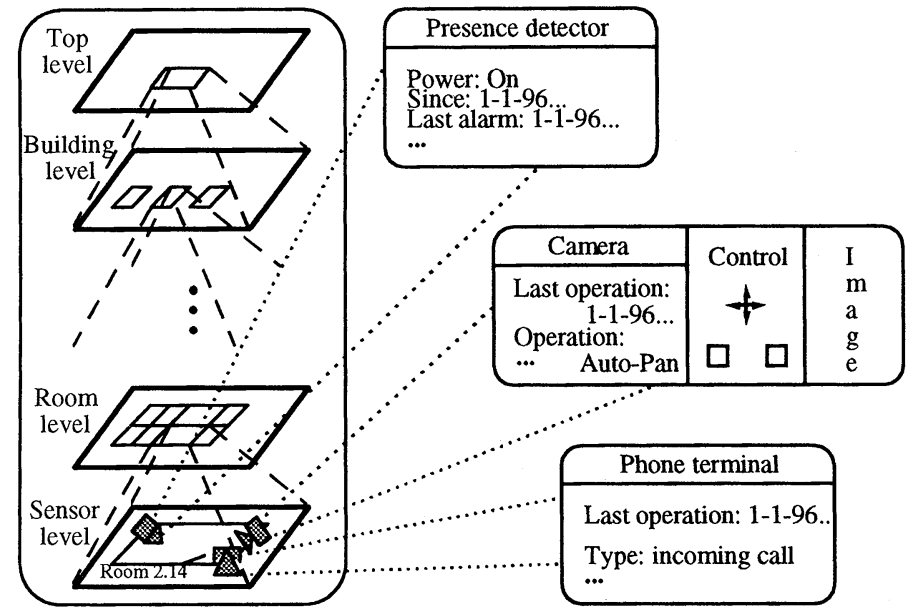

Figure 6 - Hierarchically organised synoptic.

\section{SYSTEM'S STRUCTURE OVERVIEW}

We propose that the development activities around the intelligent building concept are organised in three main groups, namely development, configuration and execution frameworks, refer to figure 7 . Those activities are associated with three completely different groups of people and computer frameworks.

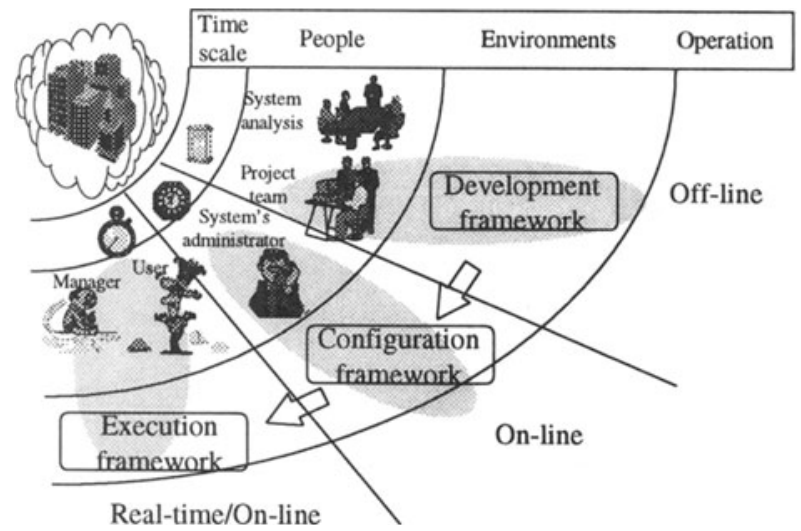

Figure 7 - Development activities around the IB concept. 
The first group to be considered, at the top level, is associated with the development framework. The goal of this stage is the design of the different systems present in the building, from architectural and construction issues to HVAC and other electronic systems subjects.

Here, different project teams are involved. From the point of view of the team associated with the "intelligent" part of the project, the main goal is to integrate information provided by different applications into the building model. They have to consider inputs from architectural $\mathrm{CAD}$, electric and electronic $\mathrm{CAD}$ and other $\mathrm{CAD}$ tools in order to represent the building in all its interesting facets. It is dangerous to consider those activities apart from the other teams, so this "intelligent" team is composed by every people that autonomously or integrated into the other project teams assure the integration of the IB concept and the cooperation between the different systems to be installed.

Between the development framework (top level, planning and design) and the floor control at the execution framework (bottom level, execution), we consider the configuration framework. The role of this framework is to download to the floor systems the designed solution.

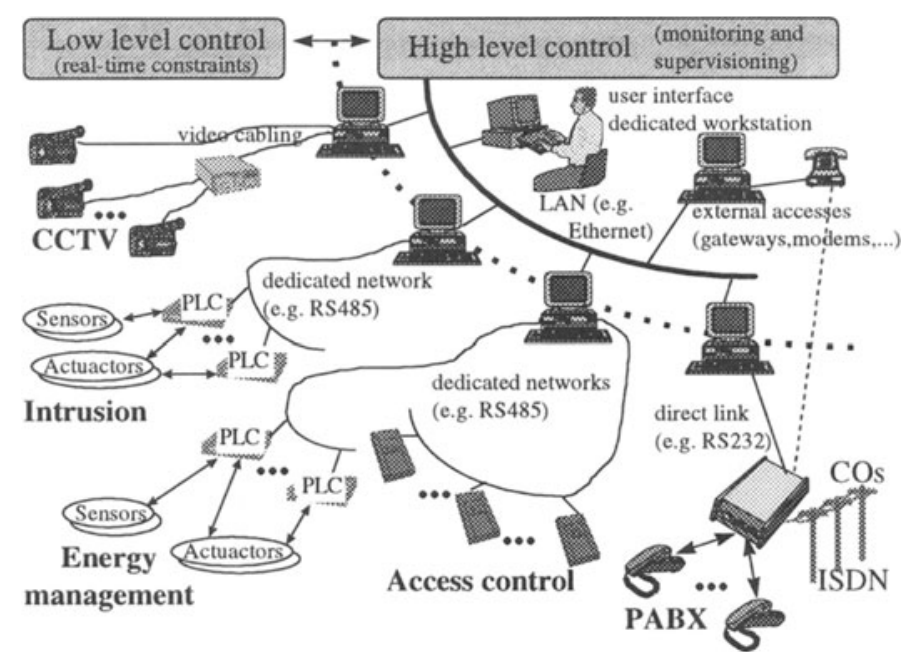

Figure 8 - Reference physical architecture for interconnection of the building systems.

Although incremental implementation is a major goal, these configuration framework is responsible for i) track of the installed versions, ii) assure easy and "soft" update between versions (from a building occupant point of view), and iii) assure easy installation of new system, functionality and activity support.

To assure that those integration activities stay coherent, we propose the use of some reference models to be used at the execution level.

The first one is related with the system architecture, from an hardware point of view. We propose a two level distributed system organisation, as shown in figure 8 . The top level is composed by monitoring workstations interconnected through a LAN, namely an Ethernet based TCP-IP LAN. The bottom level is composed by dedicated control systems, 
interconnected by some dedicated network, typically an RS-485 network. Figure 8 reproduces the infrastructure partially available and planned at one of the UNINOVA's buildings.

It is a simple and widely used reference architecture adequate for distributed system monitoring. Know-how from several areas where such architectures are used, namely manufacturing systems, can be integrated almost directly. It supports several flexibility characteristics, like incremental implementation. This means that adding new systems to the building or expand existing ones is a simple task.

The access to the information associated with some low level device is possible through the associated monitoring workstation, which means that this architecture is strongly hierarchically organised, with only two levels.

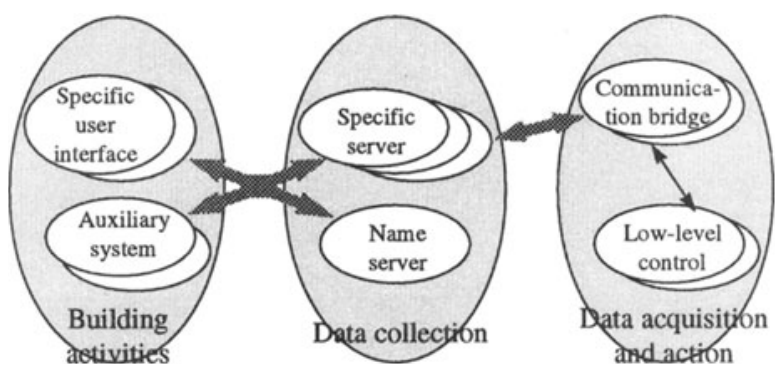

Figure 9 - Reference logical architecture for execution framework.

The second reference model proposed is related with the logical architecture, from a software point of view.

We propose a open-system distributed architecture based on different process communicating through TCP-sockets, enabling the use of heterogeneous workstations, ranging from low-cost to high-performance workstations. The bridge to low level process associated with dedicated hardware is accomplished through the use of specific gateways and protocol converters (e.g. TCP-IP-network to RS-485-network). Figure 9 shows the reference model associated with the execution framework (bottom level of figure 7).

The set of process presented in figure 9 is divided into three groups:

- building activities group, associated with the high-level control and monitoring activities and roughly identified as domots from the building occupant point of view; included in this group are user interfaces (to manage specific resources or to provide information related with different building activity analysis) and auxiliary systems (expert systems and other automatic control process responsible to autonomously supervise specific resources or emit diagnostics and suggestions to the occupants);

- data collection group, associated to the core of the monitoring systems itself and composed by a set of servers associated with specific resources to be supervised; they are responsible to provide on-line information about the status of the monitored resource and to assure the long-term storage of the related information;

- data acquisition and action group, associated with the low-level control process, responsible for action and data acquisition, running in dedicated controllers; those process are accessed through communication servers, responsible for protocol conversion (for instance, TCP-IP sockets based communication to RS-485 network). 
The referred data collection servers could be organised into two groups:

- specific servers, responsible for a specific resource management; examples include servers to manage access control systems, intrusion systems, PABX, cameras, among others;

- auxiliary servers, namely the name server, responsible to provide information related with all components of the system (devices and sub-systems), including name and associated specific server(s).

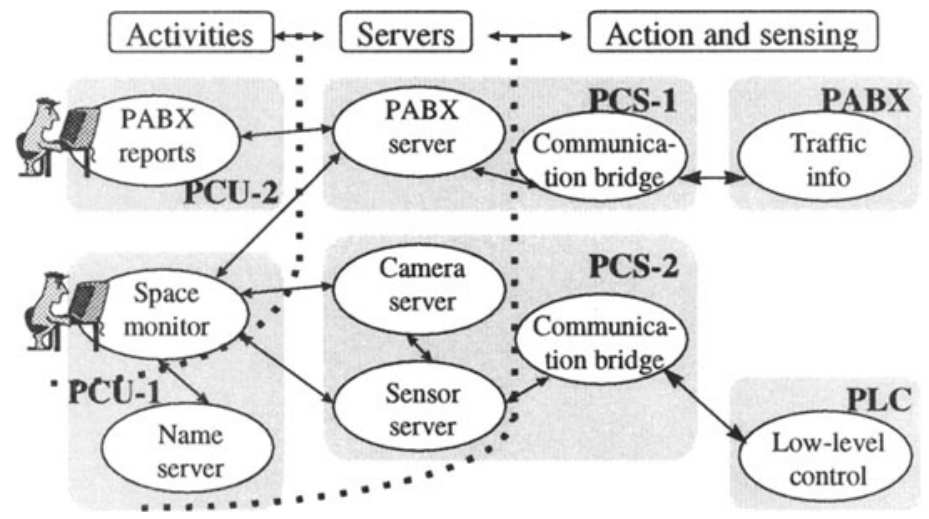

Figure 10 - Experimental set-up.

Figure 10 shows the distribution of those process over a distributed architecture composed by four workstations, one PABX and one programmable logical controller. This prototype has two user interfaces, referred by PCU-1 and PCU-2 in figure 10, the already mentioned domots at the beginning of this paper. The user interface structure of the "space monitor" domot was already presented in figure 6. Workstations PCS-1 and PCS-2 are used to support servers. The actual prototype was developed under MS-Windows for Workgroups 3.11, using Borland C++ and XVT for user interface development support tool.

\section{CONCLUSIONS}

In conclusion, we might say that solutions based on the proposed reference models for the execution environment seems to be adequate to deal and support building activities. The referred prototype is installed and ready to be operated in UNINOVA facilities in the near future.

We plan to integrate in these environment new systems, namely access control and energy management, in order to support new activities and new functionality with significant impact on the building occupant way to use the building infrastructure.

This distributed and modular architecture, enabling the definition of multiple domots, supports the cooperative integration of different results from several project teams traditionally involved in building system design.

Finally, formalisms like Petri nets with extended capabilities to integrate fuzzy control paradigms seem to be very well matched for behaviour specification, enabling hierarchical specification, simulation and automatic code generation. 


\section{ACKNOWLEDGEMENTS}

To the ISEnto project team at UNINOVA, namely Anikò Costa, João Paulo Barros, Carlos Soares and Pedro Próspero Luís and to several graduate students that contribute to earlier prototypes of parts of the system.

\section{REFERENCES}

Gomes, L. and A. Steiger-Garção (1994) Towards different buildings...; Conferencia Internacional sobre el Control Automático en el Ahorro de Energia; La Habana, Cuba; February, 21-25, 1994

Gomes, L. and A. Steiger-Garção (1995a) Petri net based Programmable Fuzzy Controller targeted for distributed control environments"; FUZZ-IEEE/IFES'95 - The Int. Joint Conference of the 4th IEEE International Conference on Fuzzy Systems and the 2nd International Fuzzy Engineering Symposium; Yokohama, Japan; March, 20-24, 1995

Gomes, L. and A. Steiger-Garção (1995b) Programmable Controllers Design based on a Synchronized Colored Petri net Model and Integrating Fuzzy Reasoning; Application and Theory of Petri Nets, 1995; Lecture Notes in Computer Science LNCS 935; Giorgio De Michelis, Michel Diaz (Eds.), pp. 218-237; Springer-Verlag; ISBN 3-540-60029-9

Harel D. (1987) Statecharts: a visual formalism for complex systems; Science of Computer Programming, 8, pp. 231-274

IBI - Intelligent Buildings Institute (1987) Intelligent Building Definition - Guideline; Public report; Washington D.C. USA

Jensen, K. (1990) Coloured Petri Nets: A High Level Language for System Design and Analysis; in Advances in Petri Nets, 1990; Lecture Notes in Computer Science; vol. 483; G. Rozenberg (ed.); Springer, Berlin; pp 342-416

Murata, T. (1989) Petri Nets: Properties, Analysis and Applications; Proceedings of the IEEE, vol. 77 , no. 4 , pp. $541-580$

\section{BIOGRAPHIES}

Luís Gomes is a lecturer and $\mathrm{PhD}$ candidate at the Electrical Engineering Department of the Faculty of Sciences and Technology of New University of Lisbon, Portugal and a researcher at UNINOVA Institute, Portugal. From 1984 to 1987, he worked in EID, Portugal, a medium enterprise, in the R\&D engineering department. His main interests include system's control modelling for discrete event systems, fuzzy control, Petri nets, statecharts and their application to sensorial architectures and intelligent buildings.

Adolfo Steiger-Garção is full professor at Department of Electrical Engineering of the Faculty of Sciences and Technology of New University of Lisbon, Portugal. He is president of UNINOVA institute and director of its Intelligent Robotics Centre. He has been responsible for several R\&D projects, involving Portuguese, European and Iberoamerican partners. He has more than 100 publications on national and international conferences and periodicals. 